\title{
Parity dependence of Nuclear Level Density
}

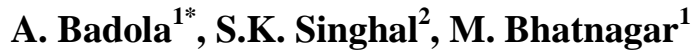 \\ ${ }^{1}$ Department of Physics, DIT University, Dehradun, India \\ ${ }^{2}$ Government Polytechnic, Saharanpur, India \\ *Corresponding Author: amitbadola555@gmail.com, Tel: +91-852-736-3420
}

Available online at: www.isroset.org

Received: 14/May/2018, Revised: 27/May/2018, Accepted: 18/Jun/2018, Online: 30/Jun/2018

\begin{abstract}
The knowledge parity dependent nuclear level density $\rho(\mathrm{E}, \mathrm{J}, \pi)$ is very essential in low energy nuclear physics as it is used to evaluate nuclear cross-sections in nuclear reaction calculations. At low excitation energies, astrophysical reaction rates are sensitive to the parity distribution. However, the information of level density is confined to a rather small region of excitation energy, angular momentum and parity. Several theoretical studies have shown that at low energies there is a dominance of one type of parity and near the particle separation energy that parity ratio only approaches to an equal distribution. An accurate determination of nuclear level density and its dependence on mass number, excitation energy, angular momentum, parity, etc. is necessary for precise prediction of cross sections using the statistical models. In the present work, an absolute asymmetry parameter is used to determine the level density of negative as well as for positive parity states by using sand $\mathrm{p}$ - wave $\left(\mathrm{D}_{0}\right.$ and $\left.\mathrm{D}_{1}\right)$ neutron level spacing. The systematic are investigated from $23<A<238$ and compared to the pattern found in previous investigations. The present analysis also provides useful information on various resonance parameters at different nuclear mass regions with results that support the original conclusions.
\end{abstract}

Keywords: Parity; Thermal and statistical models; Level density

\section{Introduction}

Description of parity-violating processes and neutroncapture reactions are important requires parity and its statistical distribution. Level densities can have considerable parity reliant as is shown by analysis of experimental data [1] and theoretical studies [2-6]. Total and parity projected level densities were calculated by using Quantum Monte Carlo methods $[7,8]$. The ratio of densities of negative and positive parity states in the neutron binding energy is usually assumed to be unity which is in accordance with some experimental results $[1-5,9,10]$. Calculations of Nakada and Alhassid [11] by employing projection method of Ref. [12] and SMMC representation of the partition function for a fixed parity clearly indicate strong parity dependence for small excitation energies. Systematic for parity distribution of nuclear level density near neutron separation energies have also been investigated by Singhal et al. [10]. Therefore, it is important to understand the dependence of nuclear level density on the key nuclear parameters like spin, parity, excitation energy etc.

According to Bethe's Fermi gas model, the nucleons were non-interacting and are proportional with the nuclear density function. In general, nuclear level density $\rho$ can be expressed as a function of various quantum numbers like excitation energy $\mathrm{U}$, angular momentum $\mathrm{J}$ and parity $\pi$ as;

$$
\rho(U, J, \pi)=F_{\pi}(U) F_{J}(U, J) \rho(U)
$$

Where,

$$
\rho(U)=\frac{1}{12 \sqrt{2} \sigma} \frac{e^{2 \sqrt{a U}}}{a^{1 / 4} U^{5 / 4}}
$$

And

$$
F_{J}(U, J)=\frac{2 J+1}{2 \sigma^{2}} e^{-J(J+1) / 2 \sigma^{2}}
$$

Here

$$
\begin{array}{ll}
\sigma^{2} & =\text { Spin cut-off parameter } \\
\mathrm{a} & =\text { Nuclear level density parameter } \\
\mathrm{U} & =\mathrm{S}_{\mathrm{n}}-\Delta=\text { Excitation Energy } \\
\Delta & =\text { Pairing energy due to odd even effect }
\end{array}
$$

As reported in the literature $[1-5,7,8,11-13]$, parity included nuclear level density function creates a large difference in the predicted theoretical cross sections. Ericson studied the parity asymmetry as a function of excitation energy. In the present study, we have determined the parity ratios at neutron binding energies for 110 nuclei between 
${ }^{23} \mathrm{Na}$ and ${ }^{238} \mathrm{U}$ by using microscopic theory. In order to take into account the energy dependence of parity distribution of nuclear level density, three parameters mainly absolute asymmetry parameter $(|\alpha|)$, absolute asymmetry parameter per nucleon $(|\alpha| / A)$ and nuclear temperature $\left(\mathrm{T}_{\mathrm{N}}\right)$ with respect to excitation energy (U) and mass number (A) curves have been plotted. A systematic study of the behavior of the parity ratio, free parameter $(\beta)$ and nuclear temperature $\left(T_{N}\right)$ across a large mass region was also performed.

Rest of the paper is organized as follows, Section I contains the introduction of parity dependence of nuclear level density; with reference from the theoretical and experimental results. Section II contain the related work of nuclear level density calculations and their analysis, Section III describes results and discussion calculated through the data, their calculations and finally from their graphs, Section IV concludes research work with future directions.

\section{Parity Ratio Analysis}

A large fraction of the level density information currently available comes from neutron resonance analysis. At low energies, the neutron can only interact with the nucleus in an $S$ state. As we move towards large excitation energies level density $(\rho)$ approaches to the value of $1 / 2$. At low energies $\rho$ is 1 for even-even nuclei. If a positive and negative parity orbit is nearly degenerate at the Fermi level than $\rho$ can be near $1 / 2$ for odd $A$ at low energy. If experiments have missed levels of a particular parity at a few $\mathrm{MeV}$, the parity ratio will not approach $1 / 2$ as the energy increases. For heavy nuclei $[14,15]$, positive and negative parity states are expected to become equal already at low excitation energy to the order of a few $\mathrm{MeV}$, while in lighter spherical nuclei stronger shell effects keep the ratio $\rho(\pi=+) / \rho(\pi=-)$ considerably different from unity over a wider energy range $[16,17]$.

Here we take the absolute asymmetry parameter $(\alpha)$ as;

$$
\alpha=\frac{D_{0}}{D_{0}+D_{1}}
$$

where, $\mathrm{D}_{0} \& \mathrm{D}_{1}$ are the s- wave and p- wave level spacing obtained from Atlas of neutron resonances [18]. An important drawback for neutron resonance data is that almost all of the available information is for $s$-wave resonances; for the large majority of neutron resonance data obtained at low neutron energies, there is limited information on $p$-wave resonances. This is because most of the neutron resonance measurements are performed at such low neutron energies. Different parity ratio have been calculated for different spin of the target nuclei but the actual formula obtained for $\alpha$ is same as in eq.(1) for all the spins as

$$
\begin{aligned}
& \alpha=\frac{3 \rho_{-}}{3 \rho_{-}+\rho_{+}} \text {and } \frac{\rho_{+}}{\rho_{-}}=\frac{3 D_{1}}{D_{0}} \text { for } I^{\pi}=0^{+} \\
& \alpha=\frac{9 \rho_{-}}{9 \rho_{-}+4 \rho_{+}} \text {and } \frac{\rho_{+}}{\rho_{-}}=\frac{9 D_{1}}{4 D_{0}} \text { for } I^{\pi}=\frac{1}{2}^{+} \\
& \alpha=\frac{2 \rho_{-}}{2 \rho_{-}+\rho_{+}} \text {and } \frac{\rho_{+}}{\rho_{-}}=\frac{2 D_{1}}{D_{0}} \text { for } I^{\pi}=\frac{3}{2}^{+}, \frac{5^{+}}{2}, \frac{7^{+}}{2} \text { and } \frac{9^{+}}{2}
\end{aligned}
$$

where, $\rho_{-} \& \rho_{+}$are the density of negative positive parity states. Value of $\alpha$ should be asymmetrically distributed about zero. From the available neutron resonance data for 110 nuclei in Atlas of Neutron Resonances [18] we obtain the best possible results. Many nuclear structure models have been employed and tested for the calculation of nuclear state densities at excitation energies corresponding to the neutron binding energy (of the order of $8 \mathrm{MeV}$ for medium and heavy nuclei). This is because at those excitation energies an important experimental quantity can be related to the density of nuclear levels; the average spacing of swave neutron resonances $\langle\mathrm{D}\rangle_{1}=0$. A large set of information on these quantities has been collected and compiled several times in the past. The data has been taken from Atlas of Neutron Resonances [18], and by using eq.(1) the values of absolute asymmetry parameter (i.e. parity ratio $(|\alpha| / \mathrm{A})$, absolute asymmetry parameter per nucleon $(|\alpha| / \mathrm{A})$ with error $(\Delta \alpha / A)$, free parameter $(\beta)$, nuclear temperature $\left(T_{N}\right)$ for 110 nuclei of positive and negative parity states has been calculated and listed in Table1 given below.

Table1. Calculated absolute asymmetry parameter $|\alpha|$, asymmetry parameter per nucleon $|\alpha| / \mathrm{A}$ with error $\Delta|\alpha| / \mathrm{A}$, free parameter

$\beta$ and Nuclear temperature $T_{N}$ values from $s$ and $p$ wave level spacings $\left(D_{0}, \Delta D_{0}, D_{1}, \Delta D_{1}\right)$ for different nuclei.

\begin{tabular}{|c|c|c|c|c|c|c|c|c|c|c|c|c|}
\hline Nucleus & $\mathbf{A}$ & $\mathbf{I}^{\boldsymbol{\pi}}$ & $\begin{array}{c}\mathbf{U}=\mathbf{S n}-\mathbf{\Delta} \\
(\mathbf{M e V})\end{array}$ & $\begin{array}{c}\mathbf{D}_{\mathbf{0}} \\
(\mathbf{k e V})\end{array}$ & $\begin{array}{c}\Delta \mathbf{D}_{\mathbf{0}} \\
(\mathbf{k e V})\end{array}$ & $\begin{array}{c}\mathbf{D}_{\mathbf{1}} \\
(\mathbf{k e V})\end{array}$ & $\begin{array}{c}\Delta \mathbf{D}_{\mathbf{1}} \\
(\mathbf{k e V})\end{array}$ & $|\boldsymbol{\alpha}|$ & $|\boldsymbol{\alpha}| \boldsymbol{A}$ & $\boldsymbol{\Delta}|\boldsymbol{\alpha}| / \boldsymbol{A}$ & $\left.\boldsymbol{\beta}_{(\mathbf{M e V}}\right)^{\mathbf{1}}$ & $\mathbf{T}_{\mathbf{N}}$ \\
\hline Na-11 & 23 & $3 / 2+$ & 6.96 & 122 & 30 & 54 & 7 & 0.69 & 0.030 & 0.009 & 0.300 & 1.739 \\
\hline Al-13 & 27 & $5 / 2+$ & 7.725 & 53 & 7 & 28.4 & 2.6 & 0.648 & 0.024 & 0.004 & 0.285 & 1.691 \\
\hline Si-14 & 28 & $0+$ & 6.206 & 332 & 35 & 109 & 11 & 0.728 & 0.026 & 0.003 & 0.346 & 1.488 \\
\hline P-15 & 31 & $1 / 2+$ & 7.937 & 54.9 & 10.4 & 21.5 & 2.2 & 0.713 & 0.023 & 0.005 & 0.280 & 1.600 \\
\hline
\end{tabular}




\begin{tabular}{|c|c|c|c|c|c|c|c|c|c|c|c|c|}
\hline S-16 & 32 & $0+$ & 6.520 & 179 & 29 & 46.3 & 4.1 & 0.768 & 0.024 & 0.005 & 0.335 & 1.427 \\
\hline S-16 & 34 & $0+$ & 4.928 & 111 & 40 & 47.5 & 4 & 0.68 & 0.020 & 0.009 & 0.463 & 1.203 \\
\hline $\mathrm{Cl}-17$ & 35 & $3 / 2+$ & 8.579 & 22.3 & 2.5 & 6.6 & 3.8 & 0.77 & 0.022 & 0.005 & 0.262 & 1.565 \\
\hline K-19 & 39 & $3 / 2+$ & 7.8 & 19.5 & 3 & 5.6 & 0.5 & 0.741 & 0.019 & 0.004 & 0.294 & 1.414 \\
\hline Ar-18 & 40 & $0+$ & 4.201 & 51.9 & 2.3 & 11.4 & 0.5 & 0.8 & 0.020 & 0.001 & 0.543 & 1.024 \\
\hline $\mathrm{Ca}-20$ & 40 & $0+$ & 6.465 & 45 & 4 & 16 & 1 & 0.72 & 0.018 & 0.002 & 0.361 & 1.273 \\
\hline Ti-22 & 46 & $0+$ & 7.110 & 20 & 3 & 6.7 & 0.5 & 0.736 & 0.016 & 0.003 & 0.337 & 1.243 \\
\hline Ti-22 & 47 & $5 / 2-$ & 11.626 & 1.64 & 0.13 & 0.47 & 0.06 & 0.752 & 0.016 & 0.001 & 0.205 & 1.572 \\
\hline Ti-22 & 48 & $0+$ & 6.409 & 20.8 & 2.5 & 8.7 & 0.87 & 0.672 & 0.014 & 0.002 & 0.382 & 1.155 \\
\hline Ti-22 & 49 & $7 / 2-$ & 10.939 & 4.52 & 0.31 & 1.5 & 0.2 & 0.735 & 0.015 & 0.001 & 0.222 & 1.494 \\
\hline $\mathrm{Cr}-24$ & 50 & $0+$ & 7.563 & 11.73 & 0.84 & 3.69 & 0.27 & 0.75 & 0.015 & 0.001 & 0.321 & 1.229 \\
\hline $\mathrm{V}-23$ & 51 & $7 / 2-$ & 7.311 & 3.95 & 0.27 & 1.7 & 0.14 & 0.663 & 0.013 & 0.001 & 0.340 & 1.197 \\
\hline $\mathrm{Cr}-24$ & 52 & $0+$ & 6.274 & 34.9 & 3.2 & 10.5 & 0.5 & 0.728 & 0.014 & 0.001 & 0.390 & 1.098 \\
\hline $\mathrm{Cr}-24$ & 53 & $3 / 2$ & 9.719 & 5.96 & 0.47 & 3.06 & 0.2 & 0.636 & 0.012 & 0.001 & 0.260 & 1.354 \\
\hline $\mathrm{Cr}-24$ & 54 & $0+$ & 4.613 & 44.3 & 5.3 & 7.72 & 0.36 & 0.81 & 0.015 & 0.002 & 0.524 & 0.924 \\
\hline $\mathrm{Fe}-26$ & 54 & $0+$ & 7.665 & 15.5 & 1 & 4.75 & 0.15 & 0.756 & 0.014 & 0.001 & 0.322 & 1.191 \\
\hline $\mathrm{Mn}-25$ & 55 & $5 / 2-$ & 7.270 & 2.42 & 0.15 & 1.1 & 0.1 & 0.66 & 0.012 & 0.001 & 0.348 & 1.149 \\
\hline $\mathrm{Fe}-26$ & 56 & $0+$ & 6.042 & 22 & 1.7 & 8.21 & 0.48 & 0.728 & 0.013 & 0.001 & 0.416 & 1.038 \\
\hline $\mathrm{Fe}-26$ & 57 & $1 / 2$ & 10.044 & 7.05 & 0.67 & 2.58 & 0.26 & 0.684 & 0.012 & 0.001 & 0.250 & 1.327 \\
\hline $\mathrm{Fe}-26$ & 58 & $0+$ & 5.005 & 21.6 & 2.6 & 5.03 & 0.3 & 0.754 & 0.013 & 0.002 & 0.495 & 0.928 \\
\hline $\mathrm{Ni}-28$ & 58 & $0+$ & 7.423 & 12.92 & 0.83 & 4.04 & 0.14 & 0.754 & 0.013 & 0.001 & 0.338 & 1.131 \\
\hline Co-27 & 59 & $7 / 2$ & 7.491 & 1.37 & 0.07 & 0.8 & 0.047 & 0.59 & 0.010 & 0.0007 & 0.348 & 1.126 \\
\hline $\mathrm{Ni}-28$ & 60 & $0+$ & 6.270 & 12.53 & 0.98 & 3.02 & 0.12 & 0.78 & 0.013 & 0.001 & 0.398 & 1.022 \\
\hline $\mathrm{Cu}-29$ & 63 & $3 / 2$ & 7.916 & 0.722 & 0.047 & 0.404 & 0.022 & 0.73 & 0.010 & 0.0009 & 0.333 & 1.120 \\
\hline $\mathrm{Ni}-28$ & 64 & $0+$ & 4.598 & 21.1 & 2 & 9.2 & 0.6 & 0.64 & 0.010 & 0.001 & 0.566 & 0.847 \\
\hline $\mathrm{Zn}-30$ & 64 & $0+$ & 6.479 & 2.94 & 0.13 & 1 & 0.05 & 0.704 & 0.011 & 0.0007 & 0.396 & 1.006 \\
\hline $\mathrm{Cu}-29$ & 65 & $3 / 2$ & 7.066 & 1.52 & 0.1 & 0.628 & 0.039 & 0.65 & 0.010 & 0.001 & 0.368 & 1.042 \\
\hline $\mathrm{Zn}-30$ & 66 & $0+$ & 5.574 & 4.7 & 0.4 & 0.84 & 0.05 & 0.792 & 0.012 & 0.001 & 0.452 & 0.919 \\
\hline $\mathrm{Zn}-30$ & 67 & $5 / 2$ & 10.198 & 0.367 & 0.019 & 0.123 & 0.005 & 0.737 & 0.011 & 0.0007 & 0.254 & 1.233 \\
\hline $\mathrm{Zn}-30$ & 68 & $0+$ & 5.026 & 3.785 & 0.19 & 1.267 & 0.037 & 0.748 & 0.011 & 0.0007 & 0.516 & 0.859 \\
\hline Ga-31 & 69 & $3 / 2-$ & 7.653 & 0.316 & 0.041 & 0.123 & 0.06 & 0.69 & 0.010 & 0.002 & 0.343 & 1.053 \\
\hline Zn-30 & 70 & $0+$ & 4.399 & 3.51 & 0.17 & 1.36 & 0.04 & $\begin{array}{l}0.7 \\
\end{array}$ & 0.010 & 0.0006 & 0.598 & 0.792 \\
\hline Ga-31 & 71 & $3 / 2-$ & 6.520 & 0.326 & 0.041 & 0.113 & 0.011 & 0.71 & 0.010 & 0.001 & 0.402 & 0.958 \\
\hline As-33 & 75 & $3 / 2$ & 7.328 & 0.093 & 0.006 & 0.054 & 0.003 & 0.6 & 0.008 & 0.0007 & 0.372 & 0.988 \\
\hline Se-34 & 76 & $0+$ & 6.042 & 0.505 & 0.065 & 0.236 & 0.033 & 0.608 & 0.008 & 0.001 & 0.447 & 0.891 \\
\hline Se-34 & 77 & $1 / 2$ & 10.497 & 0.121 & 0.011 & 0.054 & 0.005 & 0.616 & 0.008 & 0.001 & 0.257 & 1.167 \\
\hline $\mathrm{Se}-34$ & 78 & $0+$ & 5.604 & 1.48 & 0.2 & 0.515 & 0.35 & 0.702 & 0.009 & 0.002 & 0.476 & 0.847 \\
\hline $\mathrm{Br}-35$ & 79 & $3 / 2-$ & 7.892 & 0.053 & 0.002 & 0.032 & 0.003 & 0.553 & 0.007 & 0.0006 & 0.350 & 0.999 \\
\hline $\mathrm{Se}-34$ & 80 & $0+$ & 5.359 & 3.5 & 1.5 & 2.2 & 1.6 & 0.56 & 0.007 & 0.005 & 0.518 & 0.818 \\
\hline $\mathrm{Br}-35$ & 81 & $3 / 2-$ & 7.593 & 0.145 & 0.008 & 0.059 & 0.004 & 0.648 & 0.008 & 0.0007 & 0.357 & 0.968 \\
\hline $\mathrm{Kr}-36$ & 84 & $0+$ & 5.811 & 4.04 & 0.45 & 1.65 & 0.165 & 0.672 & 0.008 & 0.001 & 0.469 & 0.831 \\
\hline $\mathrm{Rb}-37$ & 85 & $5 / 2$ & 8.651 & 0.172 & 0.008 & 0.063 & 0.002 & 0.68 & 0.008 & 0.0005 & 0.314 & 1.008 \\
\hline $\mathrm{Kr}-36$ & 86 & $0+$ & 4.221 & 25.5 & 2.2 & 8.39 & 0.4 & 0.688 & 0.008 & 0.001 & 0.642 & 0.700 \\
\hline
\end{tabular}




\begin{tabular}{|c|c|c|c|c|c|c|c|c|c|c|c|c|}
\hline $\mathrm{Sr}-38$ & 87 & $9 / 2+$ & 11.113 & 0.44 & 0.07 & 0.156 & 0.011 & 0.696 & 0.008 & 0.001 & 0.245 & 1.130 \\
\hline Sr-38 & 88 & $0+$ & 5.079 & 25 & 5 & 8.7 & 1.1 & 0.704 & 0.008 & 0.002 & 0.537 & 0.759 \\
\hline Y-39 & 89 & $1 / 2-$ & 6.857 & 4.79 & 0.3 & 1.423 & 0.043 & 0.712 & 0.008 & 0.0007 & 0.396 & 0.877 \\
\hline $\mathrm{Zr}-40$ & 90 & $0+$ & 5.930 & 6.89 & 0.53 & 3.55 & 0.2 & 0.63 & 0.007 & 0.0007 & 0.472 & 0.811 \\
\hline $\mathrm{Zr}-40$ & 91 & $5 / 2+$ & 8.635 & 0.536 & 0.048 & 0.251 & 0.014 & 0.637 & 0.007 & 0.0008 & 0.323 & 0.974 \\
\hline $\mathrm{Zr}-40$ & 92 & $0+$ & 5.483 & 3.8 & 0.51 & 1.535 & 0.09 & 0.644 & 0.007 & 0.001 & 0.506 & 0.772 \\
\hline Mo-42 & 92 & $0+$ & 6.818 & 2.8 & 0.485 & 0.78 & 0.067 & 0.736 & 0.008 & 0.001 & 0.400 & 0.860 \\
\hline $\mathrm{Zr}-40$ & 93 & $5 / 2+$ & 7.228 & 0.302 & 0.075 & 0.149 & 0.015 & 0.651 & 0.007 & 0.002 & 0.388 & 0.881 \\
\hline $\mathrm{Nb}-41$ & 93 & $9 / 2+$ & 8.221 & 0.084 & 0.004 & 0.042 & 0.001 & 0.651 & 0.007 & 0.0005 & 0.342 & 0.940 \\
\hline $\mathrm{Zr}-40$ & 94 & $0+$ & 5.224 & 4 & 0.73 & 1.236 & 0.074 & 0.752 & 0.008 & 0.001 & 0.526 & 0.745 \\
\hline Mo-42 & 94 & $0+$ & 6.131 & 1.694 & 0.39 & 0.508 & 0.047 & 0.752 & 0.008 & 0.002 & 0.448 & 0.807 \\
\hline Mo-42 & 95 & $5 / 2+$ & 9.154 & 0.081 & 0.014 & 0.037 & 0.004 & 0.665 & 0.007 & 0.001 & 0.307 & 0.981 \\
\hline $\mathrm{Zr}-40$ & 96 & $0+$ & 4.350 & 13 & 4 & 4.5 & 1 & 0.672 & 0.007 & 0.003 & 0.637 & 0.673 \\
\hline Mo-42 & 96 & $0+$ & 5.596 & 1.17 & 0.28 & 0.324 & 0.025 & 0.768 & 0.008 & 0.002 & 0.491 & 0.763 \\
\hline Mo-42 & 97 & $5 / 2+$ & 8.643 & 0.046 & 0.005 & 0.021 & 0.001 & 0.679 & 0.007 & 0.001 & 0.326 & 0.943 \\
\hline Mo-42 & 98 & $0+$ & 4.712 & 0.97 & 0.2 & 0.286 & 0.014 & 0.686 & 0.007 & 0.002 & 0.586 & 0.693 \\
\hline Tc-43 & 99 & $9 / 2+$ & 6.764 & 0.012 & 0.0005 & 0.006 & 0.0003 & 0.594 & 0.006 & 0.0003 & 0.422 & 0.826 \\
\hline Mo-42 & 100 & $0+$ & 4.198 & 0.617 & 0.07 & 0.236 & 0.021 & 0.7 & 0.007 & 0.001 & 0.669 & 0.647 \\
\hline $\mathrm{Ru}-44$ & 100 & $0+$ & 5.602 & 0.447 & 0.044 & 0.115 & 0.007 & 0.7 & 0.007 & 0.001 & 0.492 & 0.748 \\
\hline $\mathrm{Ru}-44$ & 102 & $0+$ & 5.043 & 0.375 & 0.07 & 0.125 & 0.007 & 0.714 & 0.007 & 0.001 & 0.555 & 0.703 \\
\hline Rh-45 & 103 & $1 / 2$ & 6.998 & 0.028 & 0.001 & 0.013 & 0.0007 & 0.618 & 0.006 & 0.0004 & 0.408 & 0.824 \\
\hline $\mathrm{Ru}-44$ & 104 & $0+$ & 4.733 & 0.482 & 0.055 & 0.106 & 0.012 & 0.728 & 0.007 & 0.001 & 0.584 & 0.674 \\
\hline Pd-46 & 104 & $0+$ & 5.917 & 0.194 & 0.03 & 0.081 & 0.007 & 0.624 & 0.006 & 0.001 & 0.480 & 0.754 \\
\hline Pd-46 & 105 & $5 / 2+$ & 9.561 & 0.010 & 0.0005 & 0.005 & 0.0007 & 0.63 & 0.006 & 0.0005 & 0.299 & 0.954 \\
\hline Pd-46 & 106 & $0+$ & 5.370 & 0.174 & 0.025 & 0.061 & 0.004 & 0.636 & 0.006 & 0.001 & 0.526 & 0.711 \\
\hline Cd-48 & 106 & $0+$ & 6.758 & 0.135 & 0.035 & 0.052 & 0.006 & 0.636 & 0.006 & 0.002 & 0.420 & 0.798 \\
\hline Ag-47 & 107 & $1 / 2$ & 7.271 & 0.014 & 0.0005 & 0.008 & 0.0002 & 0.535 & 0.005 & 0.0003 & 0.399 & 0.824 \\
\hline Pd-46 & 108 & $0+$ & 4.999 & 0.135 & 0.024 & 0.045 & 0.003 & 0.648 & 0.006 & 0.001 & 0.566 & 0.680 \\
\hline Cd-48 & 108 & $0+$ & 6.172 & 0.12 & 0.03 & 0.048 & 0.003 & 0.648 & 0.006 & 0.002 & 0.462 & 0.755 \\
\hline Ag-47 & 109 & $1 / 2$ & 6.809 & 0.015 & 0.0006 & 0.006 & 0.0005 & 0.654 & 0.006 & 0.0004 & 0.422 & 0.790 \\
\hline Pd-46 & 110 & $0+$ & 4.581 & 0.334 & 0.043 & 0.106 & 0.009 & 0.66 & 0.006 & 0.001 & 0.618 & 0.645 \\
\hline $\mathrm{Cd}-48$ & 110 & $0+$ & 5.831 & 0.247 & 0.02 & 0.08 & 0.005 & 0.66 & 0.006 & 0.0007 & 0.486 & 0.728 \\
\hline $\mathrm{Cd}-48$ & 111 & $1 / 2+$ & 9.394 & 0.026 & 0.002 & 0.014 & 0.0009 & 0.555 & 0.005 & 0.0006 & 0.309 & 0.919 \\
\hline Cd-48 & 112 & $0+$ & 5.406 & 0.174 & 0.011 & 0.059 & 0.002 & 0.672 & 0.006 & 0.0005 & 0.527 & 0.694 \\
\hline Cd-48 & 113 & $1 / 2+$ & 9.043 & 0.024 & 0.0006 & 0.011 & 0.0006 & 0.678 & 0.006 & 0.0002 & 0.319 & 0.894 \\
\hline $\mathrm{Cd}-48$ & 114 & $0+$ & 5.017 & 0.233 & 0.03 & 0.087 & 0.008 & 0.684 & 0.006 & 0.001 & 0.572 & 0.663 \\
\hline In-49 & 115 & $9 / 2+$ & 6.785 & 0.009 & 0.0004 & 0.005 & 0.0002 & 0.575 & 0.005 & 0.0003 & 0.436 & 0.768 \\
\hline Cd-48 & 116 & $0+$ & 4.662 & 0.704 & 0.27 & 0.215 & 0.02 & 0.696 & 0.006 & 0.003 & 0.612 & 0.634 \\
\hline Sn-50 & 116 & $0+$ & 5.828 & 0.507 & 0.06 & 0.155 & 0.006 & 0.696 & 0.006 & 0.001 & 0.489 & 0.708 \\
\hline Sn-50 & 117 & $1 / 2+$ & 9.327 & 0.061 & 0.007 & 0.031 & 0.003 & 0.585 & 0.005 & 0.0008 & 0.314 & 0.892 \\
\hline Sn-50 & 118 & $0+$ & 5.379 & 0.7 & 0.15 & 0.32 & 0.04 & 0.59 & 0.005 & 0.001 & 0.542 & 0.675 \\
\hline Sn-50 & 120 & $0+$ & 5.074 & 1.485 & 0.13 & 0.378 & 0.013 & 0.72 & 0.006 & 0.0007 & 0.562 & 0.650 \\
\hline Sn-50 & 122 & $0+$ & 4.859 & 2.965 & 0.145 & 1.03 & 0.03 & 0.732 & 0.006 & 0.0003 & 0.595 & 0.633 \\
\hline
\end{tabular}




\begin{tabular}{|c|c|c|c|c|c|c|c|c|c|c|c|c|}
\hline $\mathrm{Te}-52$ & 122 & $0+$ & 5.842 & 0.146 & 0.014 & 0.063 & 0.003 & 0.61 & 0.005 & 0.0007 & 0.500 & 0.692 \\
\hline Sn-50 & 124 & $0+$ & 4.655 & 6.25 & 0.65 & 1.631 & 0.07 & 0.744 & 0.006 & 0.0008 & 0.616 & 0.612 \\
\hline Te-52 & 124 & $0+$ & 5.491 & 0.22 & 0.03 & 0.127 & 0.012 & 0.62 & 0.005 & 0.0009 & 0.543 & 0.665 \\
\hline $\mathrm{Te}-52$ & 126 & $0+$ & 5.218 & 0.64 & 0.17 & 0.247 & 0.022 & 0.63 & 0.005 & 0.001 & 0.560 & 0.643 \\
\hline $\mathrm{Te}-52$ & 128 & $0+$ & 5.021 & 1.51 & 0.375 & 0.6 & 0.077 & 0.64 & 0.005 & 0.001 & 0.585 & 0.626 \\
\hline Ba-56 & 134 & $0+$ & 5.936 & 0.36 & 0.048 & 0.12 & 0.009 & 0.67 & 0.005 & 0.0009 & 0.494 & 0.665 \\
\hline Ba-56 & 136 & $0+$ & 5.877 & 1.52 & 0.225 & 0.442 & 0.029 & 0.68 & 0.005 & 0.001 & 0.498 & 0.657 \\
\hline Ba-56 & 138 & $0+$ & 3.701 & 18.7 & 2.9 & 6.76 & 0.67 & 0.69 & 0.005 & 0.001 & 0.800 & 0.517 \\
\hline $\mathrm{Ce}-58$ & 140 & $0+$ & 4.413 & 3.73 & 0.47 & 1.55 & 0.11 & 0.7 & 0.005 & 0.0008 & 0.677 & 0.561 \\
\hline $\mathrm{Nd}-60$ & 142 & $0+$ & 5.116 & 1.035 & 0.135 & 0.39 & 0.033 & 0.71 & 0.005 & 0.0008 & 0.582 & 0.600 \\
\hline Nd-60 & 143 & $7 / 2$ & 7.817 & 0.037 & 0.002 & 0.021 & 0.004 & 0.572 & 0.004 & 0.0005 & 0.390 & 0.739 \\
\hline Nd-60 & 144 & $0+$ & 4.755 & 0.45 & 0.005 & 0.202 & 0.014 & 0.576 & 0.004 & 0.0001 & 0.634 & 0.574 \\
\hline Sm-62 & 144 & $0+$ & 5.757 & 0.77 & 0.045 & 0.265 & 0.022 & 0.72 & 0.005 & 0.0004 & 0.517 & 0.632 \\
\hline Tl-81 & 203 & $1 / 2+$ & 6.656 & 0.552 & 0.074 & 0.2 & 0.018 & 0.609 & 0.003 & 0.0006 & 0.474 & 0.572 \\
\hline $\mathrm{Pb}-82$ & 204 & $0+$ & 5.891 & 2.172 & 0.096 & 0.69 & 0.055 & 0.612 & 0.003 & 0.0002 & 0.533 & 0.537 \\
\hline Tl-81 & 205 & $1 / 2+$ & 6.504 & 2.2 & 0.3 & 1.14 & 0.08 & 0.615 & 0.003 & 0.0005 & 0.494 & 0.563 \\
\hline $\mathrm{Pb}-82$ & 206 & $0+$ & 5.901 & 37.1 & 5.5 & 4.78 & 0.21 & 0.824 & 0.004 & 0.0008 & 0.520 & 0.535 \\
\hline $\mathrm{Pb}-82$ & 207 & $1 / 2$ & 7.367 & 30 & 4 & 4.03 & 0.29 & 0.828 & 0.004 & 0.0007 & 0.417 & 0.596 \\
\hline Bi-83 & 209 & $9 / 2-$ & 4.604 & 4.54 & 0.39 & 1.11 & 0.09 & 0.627 & 0.003 & 0.0004 & 0.678 & 0.469 \\
\hline U-92 & 238 & $0+$ & 4.028 & 0.020 & 0.0007 & 0.007 & 0.0002 & 0.714 & 0.003 & 0.0001 & 0.803 & 0.411 \\
\hline
\end{tabular}

\section{Results and discussion:}

\section{A. Parity dependence of level densities}

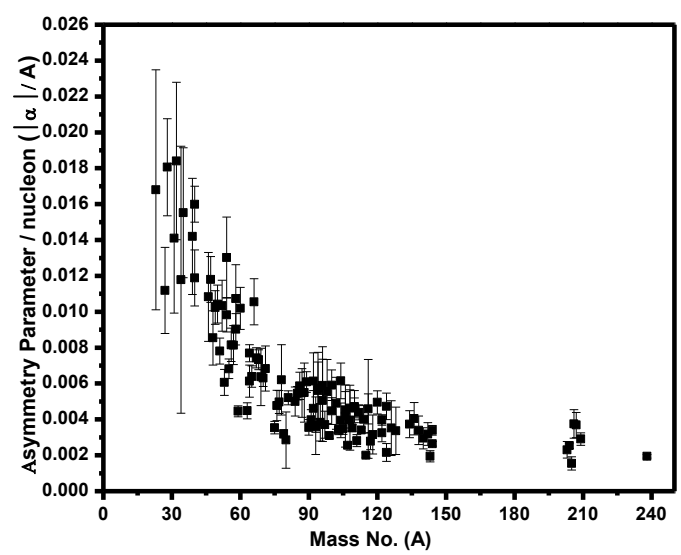

Fig1. Asymmetry parameter per nucleon $(\alpha / A)$ vs Mass number (A)

From the analysis, it has been obvious that absolute asymmetry parameter per nucleon $(|\alpha| / A)$ of compound nuclides decreases with the increase of mass number $\mathrm{A}$ and this behavior of $(|\alpha| / A)$ is due to the fact that when mass number A increases; there is a redistribution of the positive and negative parities states equally. Shell model Monte Carlo calculations $[13,14]$ in the nuclear $1 f-2 p$ shell also predict a significant parity dependence of the level density for some nuclides. The asymmetry values given by Mengoni [2] are comparable for some nuclei at low excitation energies. Values of $\rho-l \rho+$ by Allhasid [19] for Fe56, Ni-60 and $\mathrm{Zn}-68$ and Uhrenholt [20] for $\mathrm{Ni}$ and $\mathrm{Sr}$ isotopes also somehow match to the value obtained by us. The $(|\alpha| \lambda A)$ obtained from high resolution neutron resonance data by Singhal et al [10] are in agreement with our calculated values in Fig. 1. The $(|\alpha| / A)$ values for $\mathrm{Ni}, \mathrm{Sn}$ and $\mathrm{Sr}$ and some of its isotopes calculated by Mocelj [21] is in conformity with our values. Al-Quraishi [22] also calculates the parity ratio for high as well as for low excitation energy at neutron excitation energies for the nuclides on stability line which leads to equipartition of parity levels quickly at higher excitation energies. U. Agvaanluvsan [23] also determines the parity dependence of nuclear level density for different spin-parity combinations by using asymmetry parameter in high resolution proton resonance data. 


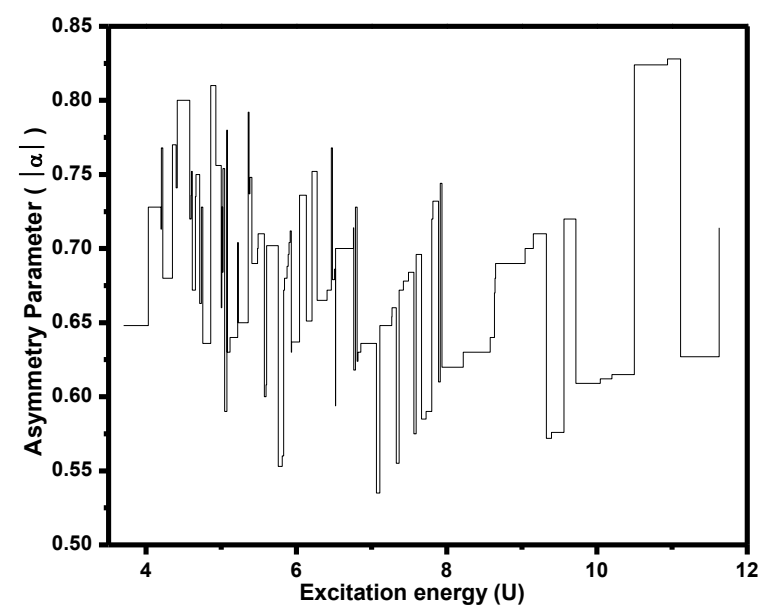

Fig2. Asymmetry parameter $(|\alpha|)$ vs. Excitation energy (U)

In fig 2 we determine the variation of absolute asymmetry parameter $(|\alpha|)$ with excitation energy (U) and it is clear from the figure that there is a variation for positive and negative parity states but as we take the magnitude of the parameter (i.e. $|\alpha|)$ that's why all variations have to be constructed on the positive side only. All the oscillations obtained between 0.5 to 0.85 i.e. close to 0.5 and 1 for all nuclei. This confirms the equiparity distribution among all the nuclei undertaken. Normally, one makes a assumption of parity equilibration as $\rho_{+}(E)=\rho_{-}(E)=1 / 2 * \rho(E)$ at all excitation energies. This is definitely not true at very low excitations. Parity ratios are only found to equilibrate up to $10 \mathrm{MeV}$ of excitation energy. However, the recent analyses [10, 24] of the experimental data indicate that equilibration is not reached even up to moderately high excitations. Allhasid [8], Rauscher [25], Mengoni [2] investigate the effect of parity distribution of excited levels on the neutron capture reactions. Positive and negative parity dominated regions in the level density has been discovered by Cerf [4] who studied the parity distribution of excited nuclear levels.

\section{B. Free parameter $(\beta)$ and Nuclear temperature $\left(T_{N}\right)$}

Now consider the energy dependence of absolute asymmetry parameter per nucleon $(|\alpha| / A)$ for the available data on neutron separation energies, we introduce a simple energy dependent function as;

$$
\frac{|\alpha|}{A}=1-\tanh \beta U
$$

In terms of nuclear temperature $\mathrm{T}_{\mathrm{N}}$,
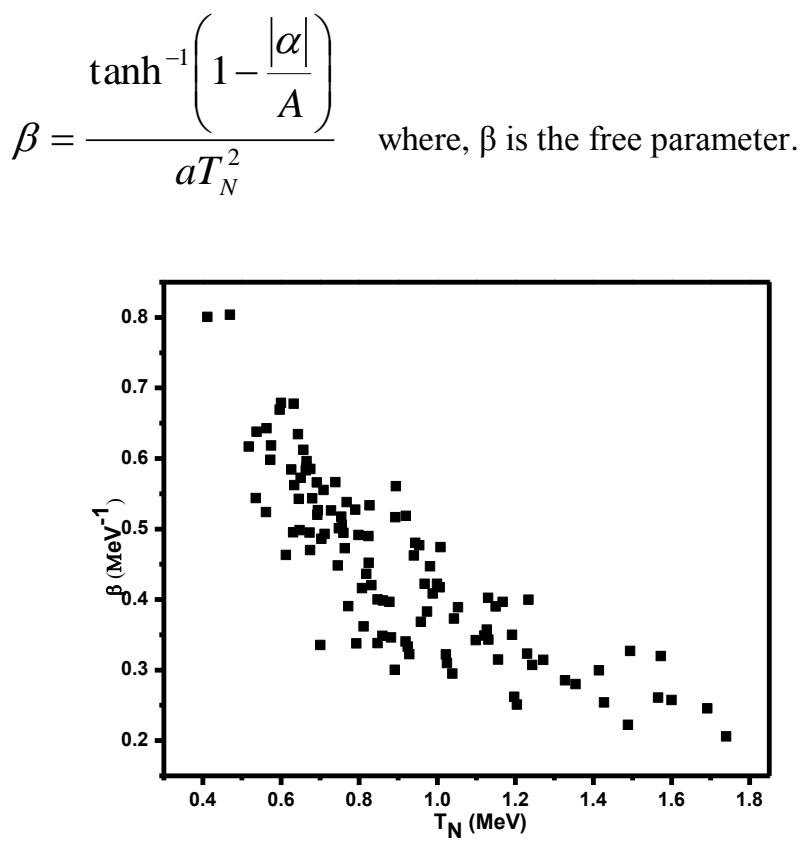

Fig3. Free Parameter $(\beta)$ vs. Nuclear Temperature $\left(\mathrm{T}_{\mathrm{N}}\right)$

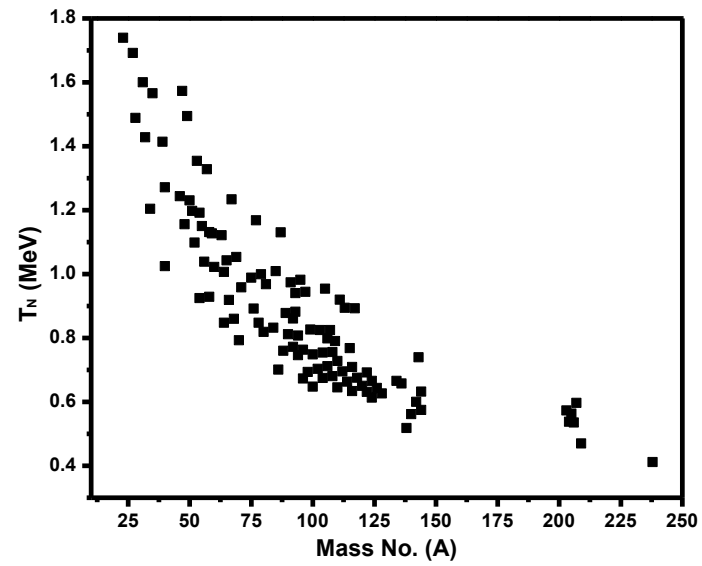

Fig4. Nuclear temperature $\left(\mathrm{T}_{\mathrm{N}}\right)$ vs. mass number $(\mathrm{A})$

At low excitation energies, with increasing nuclear temperature $T_{N}$ pairing interactions occurs in nuclear systems, leading to nucleon pairs in the ground state. The breaking of such pairs requires additional energy. Hence, at lower values of $T_{N}=1 / \beta$ the Fermi-Dirac distribution will not be able to describe the occupation properly. At high $\mathrm{T}_{\mathrm{N}}$ the microscopic distributions are well described by Fermi-Dirac statistics but for lower $T_{N}$ deviations start to appear. Free parameter $(\beta)$ as a function of nuclear temperature $\left(T_{N}\right)$ is shown in Fig3 signifying that $\beta$ decreases with the increase of $\mathrm{T}_{\mathrm{N}}$ and supports the fact of equilibrium of parities at high excitations. With increase in mass number the nuclear 
temperature $\left(\mathrm{T}_{\mathrm{N}}\right)$ decreases as shown in Fig4, which was supported by the values obtained by Gilbert et al. [26] and Kawano et al. [27].

\section{Conclusions:}

Nuclear level density is important for a variety of pure and applied nuclear physics. In this work, we have measured asymmetry parameter $(|\alpha|)$, asymmetry parameter per nucleon $(|\alpha| / A)$, free parameter $(\beta)$, nuclear temperature $\left(\mathrm{T}_{\mathrm{N}}\right)$ etc. for negative and positive parity states nuclei. A parity dependent formula has been developed to calculate the level spacing which further applied on both nuclei, giving an overall good agreement with the experimental data. From the model, the parity asymmetry can also be extracted. We have presented a simple method in the framework of the statistical Hauser-Feshbach theory to account for a full parity dependence including nonuniformly distributed parities in the nuclear level density of the compound nucleus. Further systematic study in this direction is also needed as less information is available on that.

\section{References:}

[1] B. V. Rao and H M Agrawal, "Nucl. Phys. A", Vol. 592, Issue 1, 1995.

[2] A. I. Blokhin and A. V. Ignatyuk, "Sov. J. Nucl. Phys.", Vol. 23, Issue 31, 1976.

[3] A. Mengoni, F. Fabbri, and G. Maino, "Nuovo Cimento", Vol. 94A, Issue 297, 1986.

[4] S. M. Grimes, “Phys. Rev. C”, Vol. 38, Issue 2362, 1988.

[5] N. Cerf "Nucl. Phys. A", Vol. 554, Issue 85, 1993.

[6] N. Cerf "Phys. Rev. C", Vol 49, Issue 852, 1994.

[7] G. H. Lang, C. W. Johnson, S. E. Koonin, and W. E. Ormand, "Phys. Rev. C", Vol. 48, Issue 1518, 1993.

[8] Y. Alhassid, D. J. Dean, S. E. Koonin, G. Lang and W. E. Ormand, "Phys. Rev. Lett.", Vol. 72, Issue 613, 1994.

[9] S. J. Lokitz, G. E. Mitchell and J. F. Shriner, "Phys. Rev. C”, Vol. 71, Issue 064315, 2005.

[10] S. K. Singhal and H. M. Agarwal, "Nucl. Phys. A”, Vol. 853, Issue 26, 2011.

[11] H. Nakada and Y. Alhassid, "Phys. Rev. Lett.", Vol. 79, Issue 2939, 1997.

[12] K. Tanabe, K. Sugawara-Tanabe and H. J. Mang, "Nucl. Phys. A", Vol. 357, Issue 20, 1981.

[13] D. Bucurescu and T. H. Von Egidy, “J. Phys. G: Nucl. Part. Phys.”, Vol. 31, Issue S1675, 2005.

[14] G. Maino, E. Menapace and A. Ventura "Nuovo Cimento", Vol 57A, Issue 427, 1980

[15] V. Benzi, G. Aino and E. Menapace, "Nuovo Cimento", Vol. 66A Issue 1, 1981.

[16] V. G. Soloviev, C. Stoyanov and A. I. Vdovin, "Nucl. Phys. A", Vol. 224, Issue 411, 1974.
[17] A. I. Blokhin and A. V. Ignatyuk, “Sov. J. Nucl. Phys.”, Vol. 23, Issue 31, 1976.

[18] S. F. Mughabghab, "Atlas of neutron resonances: Resonance Parameters and Thermal Cross Sections $Z=1-100$ ”, Elsevier $\left(5^{\text {th }}\right.$ ed.), New York 2006.

[19] Y. Alhassid, G. F. Bertsch, S. Liu and H. Nakada, "Phys. Rev. Lett.", Vol. 84, Issue 4313, 2000.

[20] H. Uhrenholt, S. Aberg, P. Moller and T. Ichikawa, arXiv:0901.1087v2 2009.

[21] D. Mocelj, "Phys. Rev. C”, Vol 75, Issue 045805, 2007.

[22] S. I. Al-Quraishi, S. M. Grimes, T. N. Massey and D. A. Resler, "Phys. Rev. C", Vol. 67, Issue 015803, 2003.

[23] U. Agvaanluvsan, G. E. Mitchell, J. F. Shriner and M. Pato, "Phys. Rev. C”, Vol. 67, Issue 64608, 2003.

[24] Y. Kalmykov, “Phys. Rev. Lett.”, Vol. 99, Issue 202502, 2007.

[25] T. Rauscher, "Private Communication”, 2003.

[26] A. Gilbert and A. G. W. Cameron, "Can. J. of Phys.”, Vol. 43(8), Issue 1446, 1965.

[27] T. Kawano, S. Chiba and H. Koura, “J. Nucl. Sci. Tech.”, Vol. 43(1), Issue 12006.

\section{AUTHORS PROFILE}

Mr. M Bhatnagar pursed M.Sc., M.Tech., and Ph.D. Physical Science from Meerut University, IIT, Delhi and University of Pierre et Marie Curie Paris, France in 1982, 1984 and 1992 respectively. He is currently working as Professor in Department of Physical Science in DIT University, Dehradun. He has published many research papers in reputed international journals including Elsevier and Springer (SCI \& Web of Science) and conferences.

Mr. S K Singhal pursed M.Sc. and Ph.D. Physical Science from GB Pant University, India in 1998 and 2003 respectively. $\mathrm{He}$ is currently working as Faculty of Physics, Technical Education Department, Government of Uttar Pradesh, India. He has published many research papers in reputed international journals including Elsevier and Springer (SCI \& Web of Science) and conferences. His main research work focuses on theoretical nuclear physics as well as the data ananlysis of neutron resonance parameters.

Mr A Badola pursed Bachelor of Science and Master of Science from HNB Garhwal University, India in year 2008 and 2010 respectively. He also pursed Master of Technology from JIIT University, India in year 2012. He is currently pursuing Ph.D. in Department of Physics, DITB University, India since 2013. He has published many research papers in reputed international journals including Elsevier and Springer (SCI \& Web of Science) and conferences. His main research work focuses on theoretical nuclear physics as well as the data ananlysis of neutron resonance parameters. He has 6 years of Research Experience. 